\title{
Elastografía hepática y otras secuencias avanzadas de RM (RM multiparamétrica)
}

\section{Hepatic Elastography and Other Advanced MRI Sequences (Multiparametric MRI)}

\author{
Andrés Domínguez ${ }^{1}$ Maximiliano Noceti ${ }^{1}$ Daniel Fino ${ }^{1}$ Pablo Ariza ${ }^{1}$ \\ ${ }^{1}$ Fundación Escuela de Medicina Nuclear (FUESMEN), Ciudad de \\ Mendoza, Mendoza, Argentina \\ Rev Argent Radiol 2018;82:64-71. \\ Address for correspondence Dr. Andrés Domínguez, MD, Fundación \\ Escuela de Medicina Nuclear (FUESMEN), Ciudad de Mendoza, \\ Garibaldi 405, CP: 5500, Mendoza, Argentina \\ (e-mail: andresdominguez82@gmail.com).
}

\section{Resumen \\ Palabras clave \\ - fibrosis hepática \\ - elastografía por resonancia magnética \\ - abordaje multiparamétrico}

La fibrosis es un destino donde convergen variados trastornos hepáticos. Al tratarse de un proceso dinámico y reversible, su detección temprana y una intervención terapéutica oportuna, pueden frenar su progresión. La elastografía por resonancia magnética (ERM), es un método no invasivo con notable eficacia para la valoración del grado de fibrosis hepática. La tendencia actual es combinar esa técnica con secuencias de cuantificación de lípidos y hierro, lo cual permite un abordaje multiparamétrico de los trastornos difusos del hígado.

Fibrosis is a common destination where multiple liver disorders converge. Due to its dynamic and reversible process, an early detection and timely therapeutic intervention can interrupt its progression. Magnetic Resonance Elastography (MRE) is a non-invasive method with remarkable efficacy for assessing the degree of liver fibrosis. The current trend is to combine this technique with lipid and iron quantification sequences, which allows a multiparametric approach to diffuse liver disorders.
Keywords

- hepatic fibrosis

- magnetic resonance elastography

- multiparametric approach

\section{Introducción}

La fibrosis hepática es una respuesta ante una injuria aguda o crónica del hígado por diversas causas. ${ }^{1,2}$ Consiste en un proceso de cicatrización, que tiene como fin la regeneración hepatocelular mediante activación de las células estelares, con proliferación de fibroblastos y depósito de matriz extracelular. Las bandas fibrosas que se generan distorsionan la arquitectura hepática y alteran la función del órgano. ${ }^{1}$ Tiene la particularidad de ser un proceso dinámico potencialmente reversible si se identifica y se elimina su causa. ${ }^{1,2}$ Aquí radica la importancia de su detección temprana, ya que acciones tan simples como modificar hábitos de vida en el caso del hígado

received

August 11, 2017

accepted

February 19, 2018

published online

May 14, 2018
DOI https://doi.org/

10.1055/s-0038-1641159.

ISSN 1852-9992. graso no alcohólico (HGNA) o la esteatohepatítis no alcohólica (EHNA), pueden frenar su progresión. ${ }^{3}$

Hasta hace poco tiempo, su confirmación era potestad de estudios invasivos como la biopsia percutánea o transyugular. Si bien esa continúa siendo el método de referencia, al ser costoso y no exento de complicaciones, resulta un factor limitante para confirmar el diagnóstico en muchos casos. ${ }^{4}$ Por otra parte, los biomarcadores séricos son útiles en la detección de grados avanzados de fibrosis pero tienen baja sensibilidad en diferenciar grados tempranos. ${ }^{5,6}$

La tendencia desde hace algunos años es incluir a los métodos por imágenes en la valoración de esos pacientes, siendo el más disponible en nuestro medio la elastografía de
Copyright ( 2019 , Sociedad Argentina de Radiología. Publicado por Thieme Revinter Publicações Ltda., Rio de Janeiro, Brazil. Todos los derechos reservados.

\section{License terms}

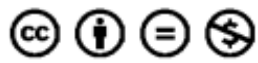


transición (ET). En la actualidad, los estudios con secuencias avanzadas de resonancia magnética (RM), han despertado un creciente interés en todo el mundo, ya que permiten una evaluación más extensa y detallada del parénquima hepático, con menos factores limitantes que los métodos de elastografía por ultrasonido (EUS) o la biopsia.

En el presente artículo, desarrollaremos el protocolo utilizado en nuestra institución, con especial énfasis en las novedosas técnicas de elastografía por resonancia magnética (ERM), cuantificación de proporción lípidos/agua (IDEAL-IQ) y cuantificación de hierro mediante secuencias ponderadas en $\mathrm{T}^{*}$.

\section{Elastografía por Resonancia Magnética}

El estudio de la fibrosis hepática es uno de los principales indicadores en la caracterización de los trastornos hepáticos más importantes que aquejan al ser humano. ${ }^{7}$ En los países industrializados las causas principales de enfermedad crónica del hígado incluyen la infección por virus de las hepatitis $\mathrm{C}$ y $\mathrm{B}$, el consumo excesivo de alcohol y trastornos vinculados a la obesidad y síndrome metabólico, como el HGNA y la EHNA. ${ }^{8}$

La ERM, es una técnica que permite valorar en forma no invasiva la rigidez de los tejidos. ${ }^{9}$ Desde sus orígenes, existe un creciente interés en todo el mundo sobre las posibles aplicaciones (próstata, cerebro, páncreas, etc.), siendo la más estudiada la valoración del grado de fibrosis hepática. 9,10

Entre las distintas escalas para cuantificar la fibrosis, se encuentra la escala METAVIR utilizada clásicamente en pacientes con hepatopatía viral crónica y hepatitis autoinmune, la cual los clasifica en 5 grupos: $\mathrm{F} 0=$ ausencia de fibrosis; $F 1=$ fibrosis leve (fibrosis en los espacios porta $\sin$ septos), F2 = fibrosis moderada (fibrosis portal con septos aislados), F3= fibrosis avanzada (extensos puentes de fibrosis que conectan los espacios porta y lobulillos) y F4= cirrosis (gruesas bandas de fibrosis con distorsión de la arquitectura y nódulos de regeneración). ${ }^{11}$ La escala de Brunt y col., ${ }^{12}$ utilizada en pacientes con EHNA los clasifica como B1 a B4, siendo la principal diferencia la distribución de la fibrosis que difiere en las distintas patologías (fibrosis portal en la primera y central en la segunda). La ERM, si bien no puede diferenciar la distribución de la fibrosis como lo hace la histopatología, si puede distinguir los distintos grados de rigidez del tejido, cuyos resultados resultan extrapolables a ambas escalas. Los grados de fibrosis por ERM se clasifican en F1: fibrosis leve, F2: fibrosis moderada, F3: fibrosis severa y F4: cirrosis. $^{9-12}$

Desde su creación, se han publicado numerosos trabajos que prueban su alto rendimiento para diferenciar hígados sanos de aquellos con fibrosis. En el consenso asiático del año 2016, donde se evaluaron los distintos métodos para valorar la fibrosis hepática, señalan a la ERM como un método recomendable para cuantificar fibrosis con nivel de evidencia $\mathrm{A} 1 .^{13}$ Un estudio retrospectivo realizado por el Dr. Yin y col., ${ }^{14}$ con 1377 pacientes, mostró una tasa de éxito de un $94,4 \%$ para diferenciar hígados con fibrosis avanzada (F3 a F4) de aquellos con fibrosis leve (F1 a F2); mientras que el meta-análisis llevado a cabo por Singh y col., ${ }^{15}$ con 697 pacientes, mostró una alta concordancia de la técnica independientemente del índice de masa corporal (IMC) y la etiología de la fibrosis, con un área bajo la curva ROC para diagnóstico de diversas etapas de fibrosis que varió de 0,84 a 0,92 (IC 95\%). Por otra parte, en una revisión sistemática de 153 estudios realizada por Bonekamp y col., ${ }^{16}$ la ERM fue el único método no invasivo capaz de estadificar con precisión el grado de fibrosis.

\section{Técnica}

- Consiste en transmitir ondas sonoras de baja frecuencia (60 Hz), hacia el interior de los tejidos y evaluar la forma en la que ellas se propagan por los mismos. ${ }^{9,10,14,15}$ Para eso se coloca sobre el abdomen del paciente un conductor circular con una membrana plástica (driver pasivo), que se encuentra conectado por medio de una manguera también plástica a una bomba neumática ubicada en la sala de máquinas (driver activo), desde la cual se genera la onda (-Fig. 1). La deformación generada en los tejidos por esas ondas mecánicas transversales o de cizallamiento, es captada por una bobina de superficie, para luego ser reconstruida mediante una secuencia sensible al movimiento (phase-contrast) y transformada mediante un software específico. Los resultados son expresados en tres formas: a) imagen en crudo, sobre la cual se hace el reproceso; b) shear waves $\mathrm{u}$ ondas de imagen, que permite una rápida referencia visual de si existió o no una adecuada propagación de la onda y c) mapas de elastograma, donde se muestra un mapa de colores de la rigidez de los tejidos, que permite comparar visualmente con la escala generada por el equipo y desde donde además se realizarán las mediciones cuantitativas utilizando la "imagen de confianza", la cual marca con un puntillado las áreas donde la medición no ha sido confiable (-Fig. 2). ${ }^{15-17}$

Para obtener los resultados se traza un ROI manualmente, evitando incluir en la muestra tanto las zonas punteadas visibles en la "imagen de confianza", como las estructuras vasculares, vesícula biliar y lesiones hepáticas que eventualmente puedan observarse (-Fig. 3). Es necesario también evitar algunos artificios propios de la técnica como los denominados "puntos calientes" o hot spots, que consisten en áreas redondeadas que simulan fibrosis avanzada, las cuales aparecen inmediatamente por debajo del driver pasivo o los generados en el contorno hepático, conocidos como edge artifact. $^{18}$ Los resultados son expresados en kilopascales ( $\mathrm{kPa}$ ) y comparados con valores de referencia, ${ }^{18}$ donde se considera:

- Valor Normal: menor a $2.5 \mathrm{kPa}$.

- Normal/Inflamación crónica: 2.5 a 2.9 kPa.

- Fibrosis leve (F1 a F2): 2.9 a $3.5 \mathrm{kPa}$.

- Fibrosis moderada (F2 a F3): 3.5 a $4 \mathrm{kPa}$.

- Fibrosis avanzada (F3 a F4): 4 a $5 \mathrm{kPa}$

- Cirrosis (F4): mayor a $5 \mathrm{kPa}$. 

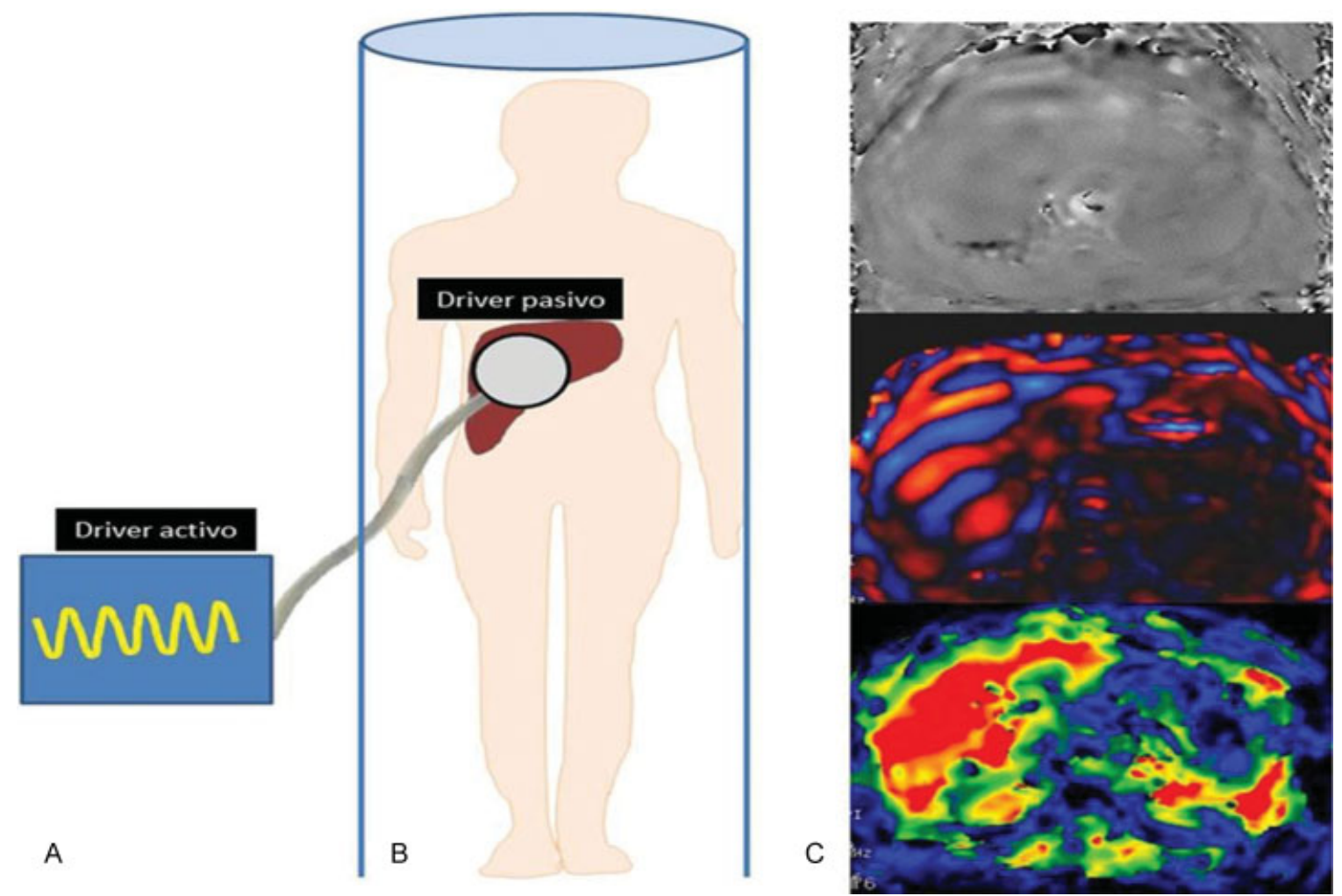

Fig. 1 Gráfico que muestra: (A) Driver activo ubicado en la sala de máquinas, el cual genera la onda mecánica; (B) esa onda se transmite por medio de una manguera plástica hacia el driver pasivo, el cual se coloca sobre la pared abdominal. (C) Imágenes obtenidas: imagen phase-contrast cruda (arriba), shear waves u ondas de imagen (al medio) y mapa de elastograma (abajo). Las imágenes en color rojo en el mapa de elastograma representan áreas de fibrosis avanzada (F4), en un paciente de 47 años en control por VHC.

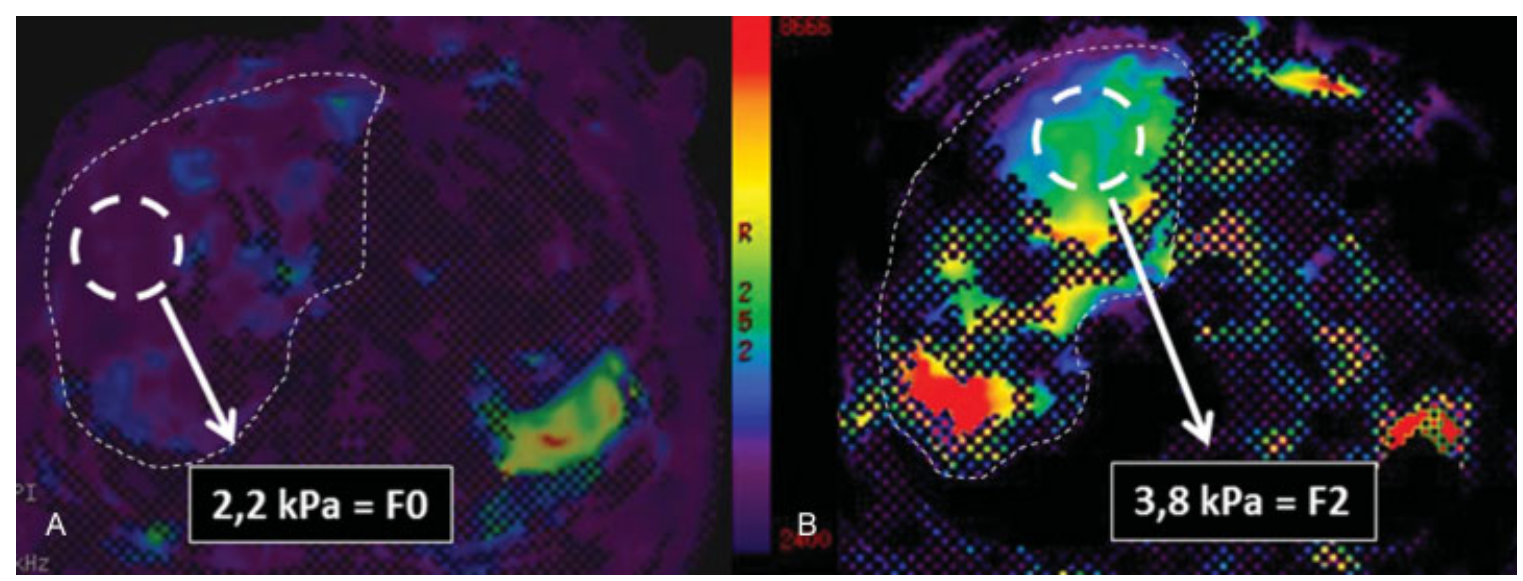

Fig. 2 Elastografía por resonancia magnética (ERM) imagen de confianza, que muestra un puntillado en color negro, el cual debemos evitar al trazar el ROI. (A) Paciente voluntario sano, donde la rigidez del hígado se asemeja a la del TCS, y el análisis cuantitativo arroja una rigidez de 2,2 kPa, compatible con un F0. (B) Paciente enolista crónico donde vemos un área en el segmento lateral sin puntillado apta para la medición, la cual fue de 3,8 kPa, compatible con una fibrosis moderada (F2 a F3).

\section{Ventajas}

- Algunas de las ventajas de la ERM respecto de la EUS y la biopsia, es que la primera evalúa áreas mucho mayores de tejido, ya que de todos los equipos de EUS disponibles en el mercado, el que mayor alcance tiene puede evaluar hasta $20 \mathrm{cc}$, mientras que en la ERM se evalúan aproximadamente 250 cc. $^{19}$ Ese hecho es crucial si tenemos en cuenta que la fibrosis puede no ser homogénea, por lo cual, mientras más cantidad de tejido evaluemos, más correlación habrá con el estado real del hígado del paciente (-Fig. 4). ${ }^{20}$ Eso la convierte también en una referencia útil, que puede servir de guía en la toma de la biopsia.

Permite además, evaluar la respuesta al tratamiento, hecho que limita a la biopsia por tratarse de un método invasivo y 


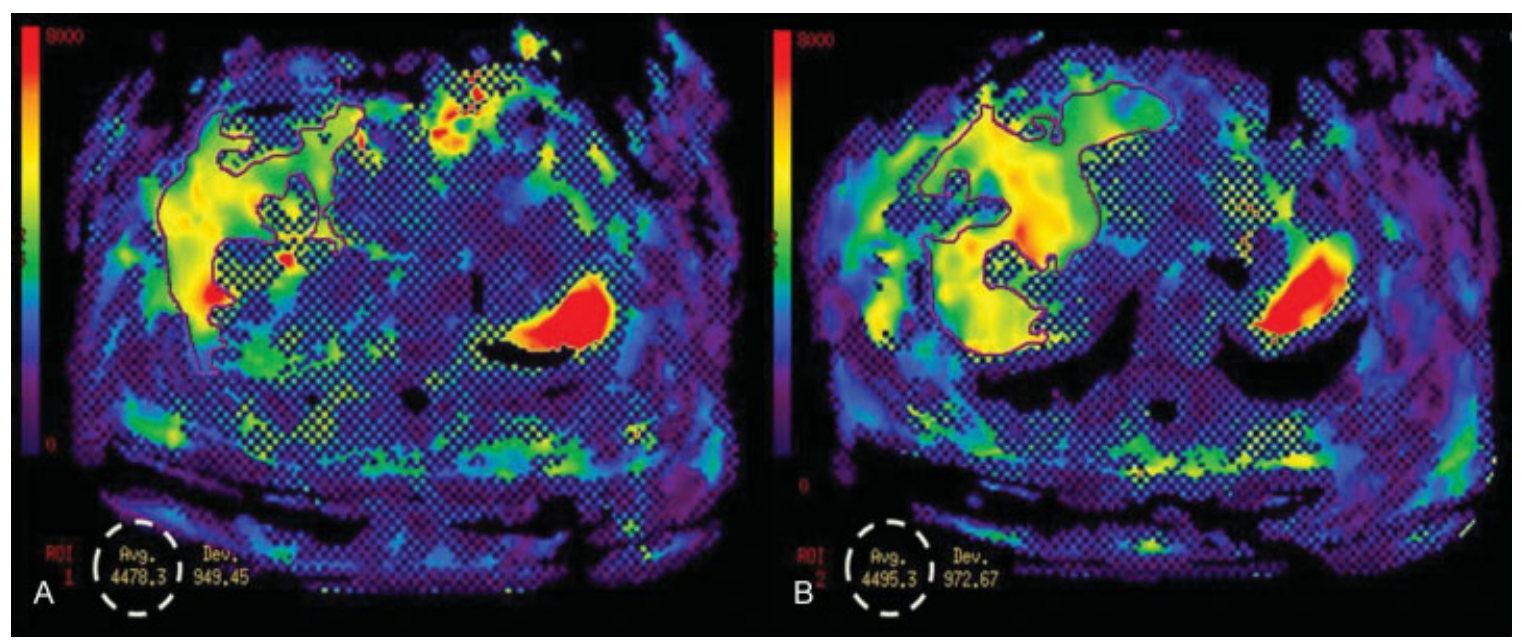

Fig. 3 (A y B) Muestran el ROI de trazado manual evitando tanto el puntillado de la imagen de confianza, como estructuras vasculares, vesícula biliar y artificios propios de la técnica. En ese caso, y con el círculo punteado, se señala la rigidez promedio en esos 2 cortes, la cual es de aproximadamente 4,4 kPa, compatible con una fibrosis avanzada (F3 a F4).

supera a la EUS por ser ese un método operador dependiente, siendo por ende menos reproducible que la ERM. ${ }^{21}$ Otra de las ventajas es que la obesidad no altera los resultados, importante si tenemos en cuenta la creciente relación de la obesidad y el síndrome metabólico como causales de enfermedad crónica hepática, vinculada a HGNAy EHNA. Eso último la distingue de la EUS, donde es conocida la limitación que genera la obesidad en la conducción del ultrasonido. ${ }^{19,21}$ Cabe destacar además que los resultados tampoco se ven alterados por grados leves a moderados de ascitis, la cual puede ser un factor limitante en la toma de biopsia percutánea. ${ }^{22}$

Son ventajas adicionales de la RM la detección de complicaciones de la cirrosis como el HCC si lo combinamos con secuencias convencionales, además de la posibilidad de cuantificar hierro y proporción de lípidos durante el mismo estudio, sin incrementar significativamente el tiempo de exploración (- Fig. 5). ${ }^{23}$

\section{Limitaciones}

- Una de las principales es la sobrecarga moderada a severa de hierro, ya que ella altera la relación señal/ruido (SNR) y puede generar errores en la medición, no dejando zonas medibles en la imagen de confianza. ${ }^{24}$ Es por ese motivo, que consideramos conveniente incluir la cuantificación de hierro en este protocolo. Cabe señalar que para esos casos disponemos de otras técnicas de adquisición de la ERM basadas en secuencias eco gradiente, que permiten contrarrestar al menos en parte ese efecto.

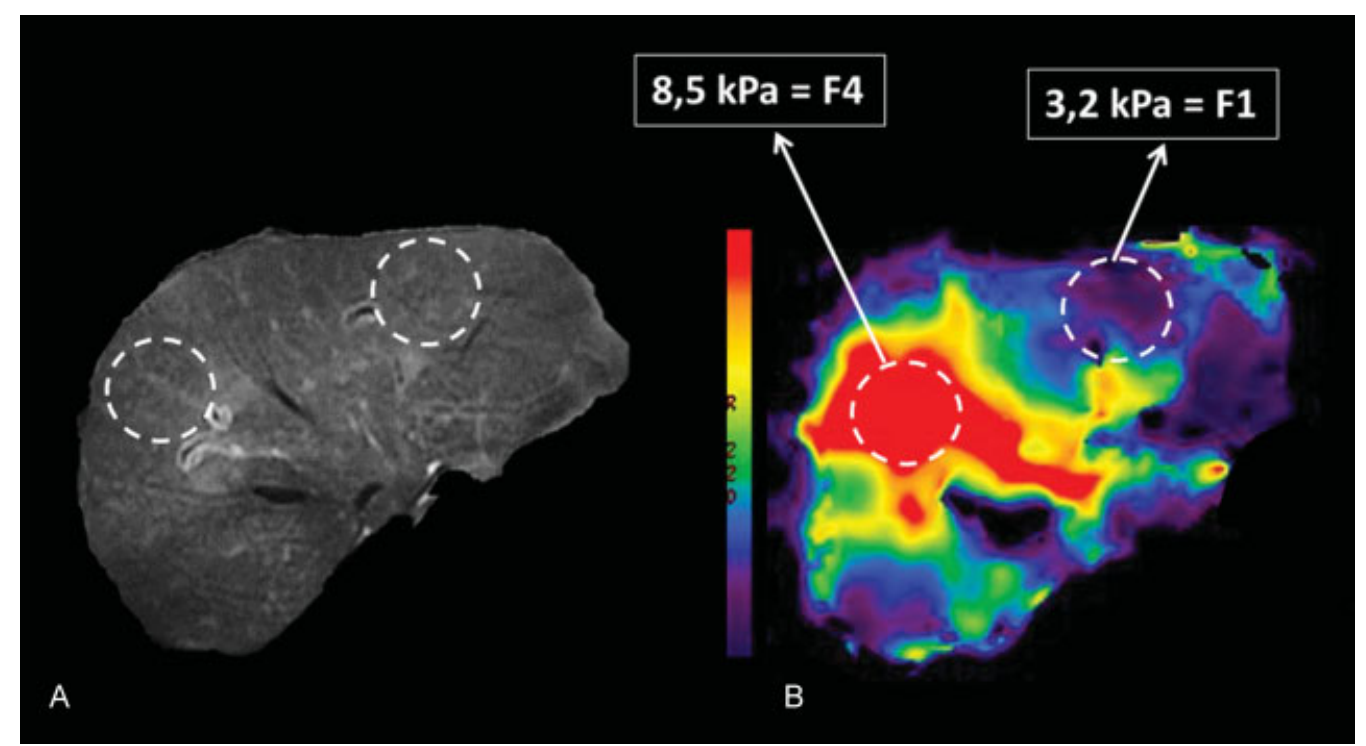

Fig. 4 (A y B) Axial ponderado en T2 y ERM en un paciente en control por VHC. Nótese la distribución heterogénea de la fibrosis, que es avanzada hacia los segmentos $\mathrm{V}$ y VIII $(8,5 \mathrm{kPa})$ y se hace progresivamente menor hacia el segmento lateral $(3,5 \mathrm{kPa})$ y segmentos posteriores. Una eventual toma de biopsia de los segmentos menos afectados, hubiese infraestimado la enfermedad. 


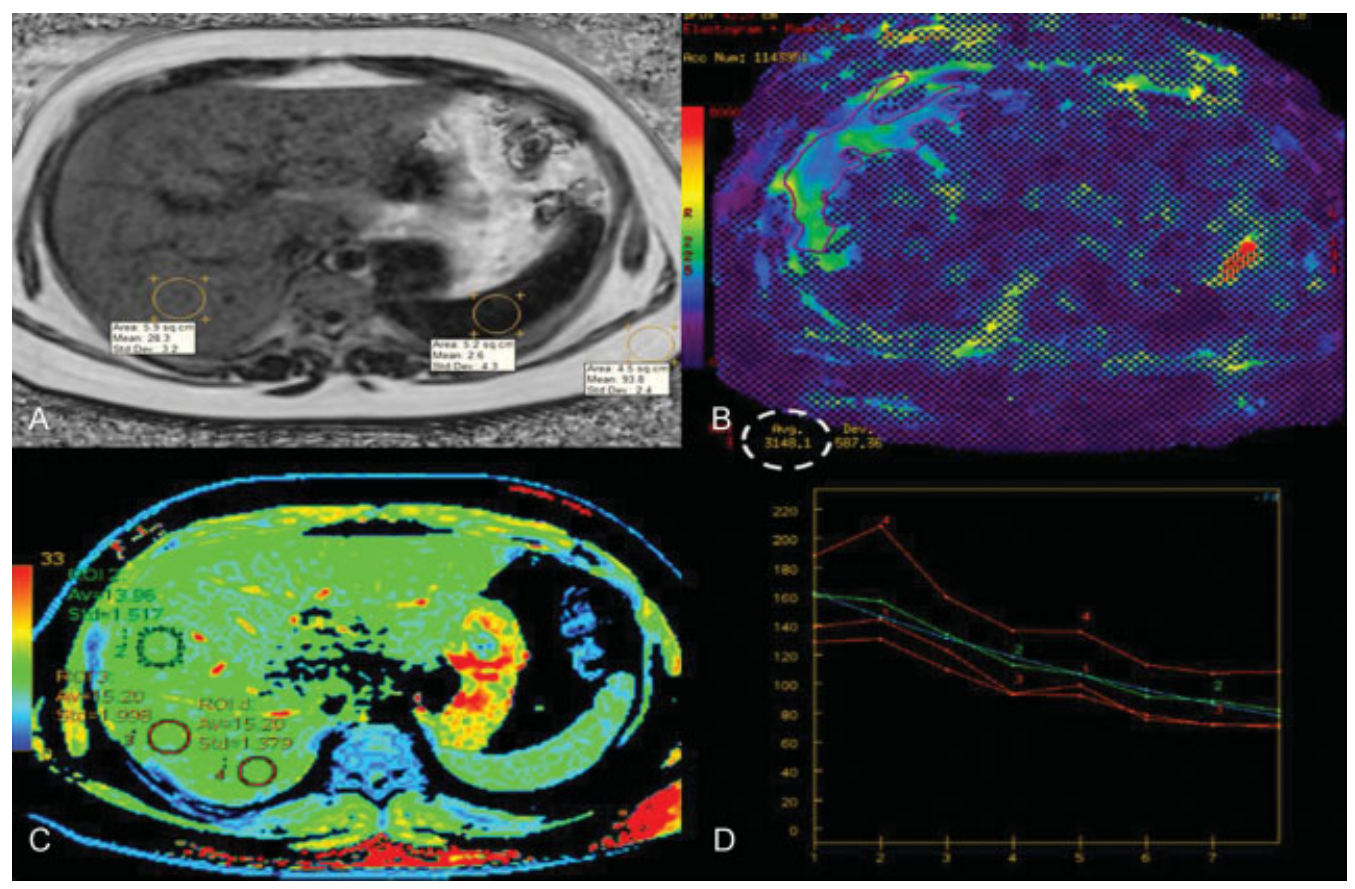

Fig. 5 Estudio multiparamétrico hepático en un paciente de 19 años en tratamiento con antiretrovirales. (A) Secuencia DIXON que muestra una proporción de lípidos de $27 \%$ por esteatosis severa. (B) ERM con leve incremento en la rigidez (3,1 kPa), probablemente en contexto de una EHNA. (C y D) Técnicas mapping que muestra un valor T2* de 15 . El cálculo de la concentración hepática de hierro da un valor de $1,9 \mathrm{mg} / \mathrm{g}$, encontrándose dentro de los parámetros normales.

En un estudio reciente se señala, además de la sobrecarga de hierro, a la ascítis masiva como un factor limitante, encontrando mayores fallas en la medición de los estudios realizados con equipos de $3 \mathrm{~T}$ comparado con los de $1.5 \mathrm{~T} .^{25}$

Entre las limitaciones vinculadas a la técnica, se destaca que al tratarse de una secuencia adquirida durante apnea espiratoria, se requiere de colaboración por parte del paciente. Por otra parte, la falta de ayuno puede sobrestimar (en forma leve) el grado de rigidez debido al aumento del flujo venoso portal, por lo que se requiere de un ayuno total de al menos 6 horas. $^{26}$

Además, si bien como se dijera anteriormente la obesidad no altera las mediciones, el tamaño del tubo y el ingreso del paciente por el mismo es un factor excluyente. Otras limitaciones son comunes a los estudios de RM como marcapasos o reemplazo valvular mecánico no compatibles, implantes cocleares, etc.

La rigidez del hígado puede verse levemente incrementada por causas como la inflamación crónica, hipertensión portal y hepatopatía congestiva, mientras que la inflamación aguda y la amiloidosis pueden incrementarla en forma significativa, representando no necesariamente una desventaja, pero si un factor a tener en cuenta durante la interpretación del estudio. ${ }^{18}$ En la actualidad, se encuentran en investigación nuevas técnicas de elastografía tridimensional, que podrían permitir diferenciar entre diferentes causas de incremento en la rigidez.

Por último, al tratarse de una tecnología relativamente nueva en nuestro medio, tiene menor alcance y es más costosa que la EUS, aunque la posibilidad de la ERM de cuantificar lípidos y hierro en el mismo examen puede compensar ese último hecho. ${ }^{23}$

\section{Posibles horizontes}

- Diversos estudios señalan la utilidad de la ERM en el campo de la oncología. Uno de ellos menciona la ERM como un biomarcador de respuesta temprana, donde las modificaciones en la rigidez del tejido tumoral en respuesta al tratamiento precede tanto a los cambios en el tamaño tumoral, como a los de otros biomarcadores como la difusión (celularidad) o la perfusión (neovascularización). ${ }^{27}$ Otro estudio reciente mostró una diferencia significativa en el grado de rigidez del HCC tratado (RE y TACE/RF), comparado con los no tratados, siendo menor que en los primeros. ${ }^{28}$ Por otra parte, se cree que puede tener utilidad en la diferenciación de tumores hepáticos benignos versus malignos, donde en esos últimos, la rigidez será mayor. ${ }^{29}$

\section{Proporción Lípidos (Proton Density Fat Fraction)}

Las técnicas para la cuantificación de lípidos constituyen también un avance importante en el manejo del paciente con enfermedad difusa hepática. Varios estudios prueban la creciente incidencia del HGNA y EHNA como causales de enfermedad crónica hepática vinculados a la obesidad y síndrome metabólico, trastornos que alcanzan proporciones epidémicas en países industrializados. ${ }^{30,31}$ Por eso la importancia de sumar a los estudios de ERM secuencias que permitan valorar lípidos.

Entre las distintas técnicas por imágenes para la cuantificación de lípidos, utilizamos la secuencia DIXON single breath hold multipoint (IDEAL-IQ; GE), que permite 
valorar la proporción de lípidos en densidad protónica sin incrementar significativamente el tiempo de exploración $(<$ 2 minutos). ${ }^{32,33}$ Ese hecho la distingue de la espectroscopia, la cual aumentaría significativamente el tiempo de adquisición, permitiéndonos de esa manera, incluir a la técnica IDEAL-IQ en el protocolo de rutina de los trastornos hepáticos difusos. Un porcentaje menor a 6\% es considerado normal, siendo el punto de corte de $15 \%$ para diferenciar grados leves de moderados a severos. ${ }^{33}$

Estudios recientes plantean la posibilidad de distinguir entre HGNA de EHNA mediante RM al combinar las técnicas de valoración de lípidos con la ERM, donde la rigidez del tejido hepático no se ve alterada en el primero, mientras que se encuentra incrementada en el segundo (- Fig. 6). ${ }^{34,35}$

\section{Cuantificación de Hierro con Secuencias Ponderadas en T2* (Técnicas Mapping)}

Para la cuantificación de la concentración hepática de hierro (LIC), utilizamos una secuencia multiecho gradient-recalled echo, que proporciona el valor $\mathrm{T} 2{ }^{*}$ en milisegundos. ${ }^{36}$ Eso permite calcular el coeficiente de relaxometría R2* (1000/ $\mathrm{T}^{*}$ ) en unidades de segundo, para posteriormente mediante un algoritmo matemático $\left[\left(0.0254 \times \mathrm{R} 2^{*}\right)+0.202\right]$, obtener la LIC, cuyo resultado se expresa en miligramo de hierro por cada gramo de tejido hepático seco $(\mathrm{mg} / \mathrm{g}) .{ }^{37}$ Se considera valor aceptable $<3.5 \mathrm{mg} / \mathrm{g}$; sobrecarga leve 3.5 a $7 \mathrm{mg} / \mathrm{g}$; moderada 7 a $12 \mathrm{mg} / \mathrm{g}$ y severa $>12 \mathrm{mg} / \mathrm{g}$ (-Fig. 7). ${ }^{36}$ Ese método maximiza la relación señal/ruido (SNR) ya que no depende de una referencia interna (anatómica) ni externa para determinar el R2 o el R2*, mostrando una fuerte correlación con la LIC obtenida en biopsias hepáticas. ${ }^{38-40}$ Cabe aclarar que esas técnicas requieren de un software específico y no están disponibles en forma masiva, en cuyo caso puede utilizarse también el método SIR (signal intensity ratio), ampliamente utilizado en todo el mundo (método de la Universidad de Rennes), que compara la intensidad de señal del hígado con la de los músculos paraespinales. ${ }^{41}$

\section{Protocolo de Evaluación de los Trastornos Hepáticos Difusos}

Todas las imágenes fueron adquiridas con un equipo Signa PET-RM 3T (GE Healthcare).

El protocolo completo consistió en cortes axiales y coronales ponderados en T2 single shot fast spin eco (SSFSE), secuencias de desplazamiento químico en axial y las secuencias avanzadas: ERM, IDEAL-IQ y secuencia ponderada en $\mathrm{T}^{*}$ para la cuantificación del hierro. El tiempo neto de adquisición fue de aproximadamente 15 minutos, con un promedio entre el ingreso del paciente al equipo y su salida menor a 25 minutos.

\section{Conclusión}

La ERM es un método idóneo para cuantificar la fibrosis hepática. Sumado a las secuencias avanzadas para valorar lípidos y hierro, permite una evaluación no invasiva $\mathrm{y}$

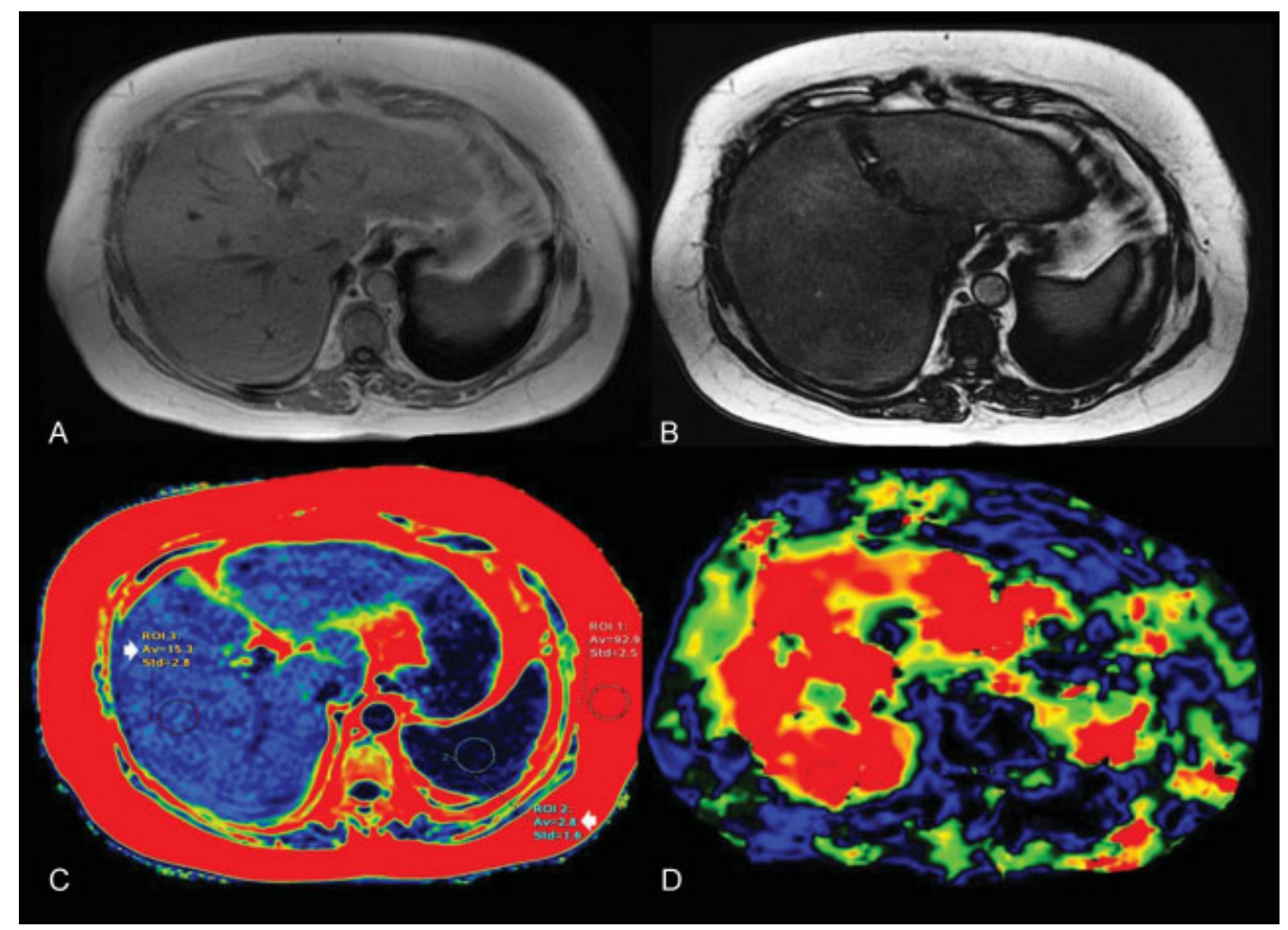

Fig. 6 Paciente de 55 años con síndrome metabólico y aumento de enzimas hepáticas de causa no aclarada. (A y B) Secuencias de desplazamiento químico que muestran una caída de la señal en la secuencia en fase opuesta, por esteatosis. (C) Secuencia DIXON (IDEAL-IQ), que muestra una proporción de lípidos de 15\% comparado con la del bazo ( $<5 \%$ ), por esteatosis moderada/severa. (D) ERM con marcado aumento en la rigidez del tejido hepático (8,2Kpa), hallazgos compatibles con una esteatohepatítis no alcohólica (EHNA) asociada a fibrosis avanzada (F4). 

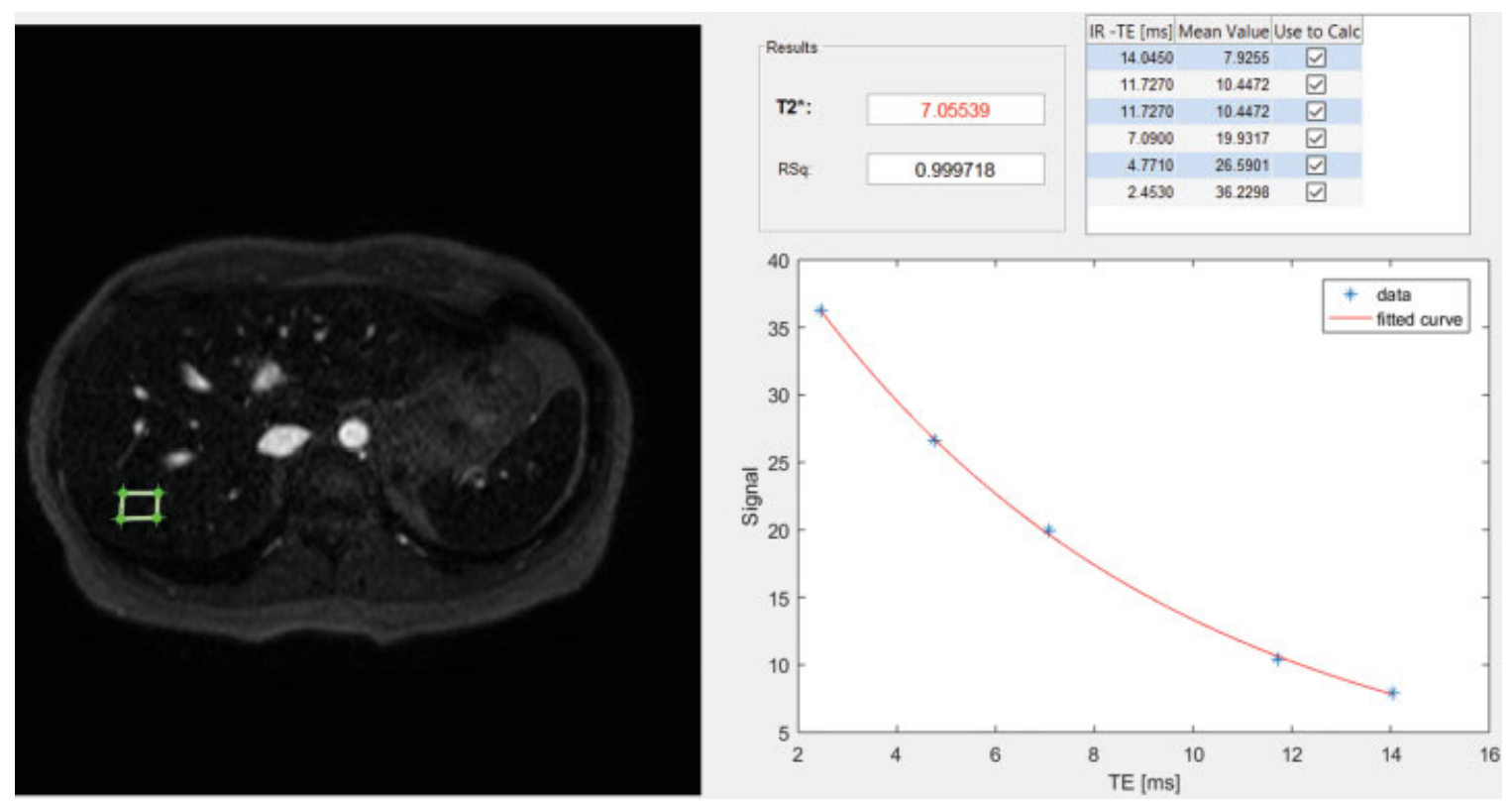

Fig. 7 Paciente de 38 años con antecedentes de talasemia. La cuantificación de hierro en ese paciente arroja un valor T2* de 7.05, siendo la concentración hepática de hierro de $3.8 \mathrm{mg} / \mathrm{g}$, compatible con una sobrecarga leve. El gráfico de la izquierda muestra la caída progresiva de la señal en función del incremento del TE, lo que permite cuantificar el T2*, necesario para obtener el coeficiente de relaxometría y posteriormente la LIC.

multiparamétrica de la enfermedad hepática difusa, aplicable a la práctica clínica. Si bien su uso resulta limitado en la actualidad debido a la baja disponibilidad en nuestro medio, el advenimiento de las nuevas tecnologías obliga al radiólogo a estar familiarizado con esas técnicas.

\section{Responsabilidades Éticas}

Protección de personas y animales. Los autores declaran que para esta investigación no se han realizado experimentos en seres humanos ni en animales.

Confidencialidad de los datos. Los autores declaran que han seguido los protocolos de su centro de trabajo sobre la publicación de datos de pacientes.

Derecho a la privacidad y consentimiento informado. Los autores declaran que en este artículo no aparecen datos de pacientes.

\section{Conflicto de Intereses}

Los autores declaran no tener ningún conflicto de interés, excepto el Dr. Domínguez que declara como posible conflicto de interés ser revisor de la Revista Argentina de Radiología.

\section{Bibliografía}

1 Wallace K, Burt AD, Wright MC. Liver fibrosis. Biochem J 2008;411 (01):1-18

2 Bataller R, Brenner DA. Liver fibrosis. J Clin Invest 2005;115(02): 209-218

3 Younossi ZM, Stepanova M, Rafiq N, et al. Pathologic criteria for nonalcoholic steatohepatitis: interprotocol agreement and ability to predict liver-related mortality. Hepatology 2011;53(06): $1874-1882$
4 Piccinino F, Sagnelli E, Pasquale G, Giusti G. Complications following percutaneous liver biopsy. A multicentre retrospective study on 68,276 biopsies. J Hepatol 1986;2(02):165-173

5 Huwart L, Sempoux C, Salameh N, et al. Liver fibrosis: noninvasive assessment with MR elastography versus aspartate aminotransferase-to-platelet ratio index. Radiology 2007;245(02):458-466

6 Castera L. Invasive and non-invasive methods for the assessment of fibrosis and disease progression in chronic liver disease. Best Pract Res Clin Gastroenterol 2011;25(02):291-303

7 Fassio E, Mazzolini G, Villamil A, Gadano A. Consenso argentino de hepatocarcinoma. 2015. Disponible en: http://www.aaeeh.org. ar/consensos-y-guias/. (consultado Feb 2018)

8 Younossi ZM, Stepanova M, Afendy M, et al. Changes in the prevalence of the most common causes of chronic liver diseases in the United States from 1988 to 2008. Clin Gastroenterol Hepatol 2011;9(06):524-530.e1, quiz e60

9 Muthupillai R, Lomas DJ, Rossman PJ, Greenleaf JF, Manduca A, Ehman RL. Magnetic resonance elastography by direct visualization of propagating acoustic strain waves. Science 1995;269(5232):1854-1857

10 Venkatesh SK, Yin M, Ehman RL. Magnetic resonance elastography of liver: clinical applications. J Comput Assist Tomogr 2013;37 (06):887-896

11 Bedossa P, Poynard T; The METAVIR Cooperative Study Group. An algorithm for the grading of activity in chronic hepatitis $C$. Hepatology 1996;24(02):289-293

12 Brunt EM, Janney CG, Di Bisceglie AM, Neuschwander-Tetri BA, Bacon BR. Nonalcoholic steatohepatitis: a proposal for grading and staging the histological lesions. Am J Gastroenterol 1999;94 (09):2467-2474

13 Shiha G, Ibrahim A, Helmy A, et al. Asian-Pacific Association for the Study of the Liver (APASL) consensus guidelines on invasive and non-invasive assessment of hepatic fibrosis: a 2016 update. Hepatol Int 2017;11(01):1-30

14 Yin M, Glaser KJ, Talwalkar JA, Chen J, Manduca A, Ehman RL. Hepatic MR elastography: clinical performance in a series of 1377 consecutive examinations. Radiology 2016;278(01):114-124 
15 Singh S, Venkatesh SK, Wang Z, et al. Diagnostic performance of magnetic resonance elastography in staging liver fibrosis: a systematic review and meta-analysis of individual participant data. Clin Gastroenterol Hepatol 2015;13(03):440-451.e6

16 Bonekamp S, Kamel I, Solga S, Clark J. Can imaging modalities diagnose and stage hepatic fibrosis and cirrhosis accurately? J Hepatol 2009;50(01):17-35

17 Rustogi R, Horowitz J, Harmath C, et al. Accuracy of MR elastography and anatomic MR imaging features in the diagnosis of severe hepatic fibrosis and cirrhosis. J Magn Reson Imaging 2012;35(06):1356-1364

18 Srinivasa Babu A, Wells ML, Teytelboym OM, et al. Elastography in chronic liver disease: modalities, techniques, limitations, and future directions. Radiographics 2016;36(07):1987-2006

19 Barr RG, Ferraioli G, Palmeri ML, et al. Elastography assessment of liver fibrosis: Society of radiologists in ultrasound consensus conference statement. Radiology 2015;276(03):845-861

20 Marcellin P, Asselah T, Boyer N. Fibrosis and disease progression in hepatitis C. Hepatology 2002;36(05, Suppl 1):S47-S56

21 Huber A, Ebner L, Heverhagen JT, Christe A. State-of-the-art imaging of liver fibrosis and cirrhosis: A comprehensive review of current applications and future perspectives. Eur J Radiol Open 2015;2:90-100

22 Grant A, Neuberger J; British Society of Gastroenterology. Guidelines on the use of liver biopsy in clinical practice. Gut 1999;45(Suppl 4):IV1-IV11

23 Banerjee R, Pavlides M, Tunnicliffe EM, et al. Multiparametric magnetic resonance for the non-invasive diagnosis of liver disease. J Hepatol 2014;60(01):69-77

24 Taouli B, Ehman RL, Reeder SB. Advanced MRI methods for assessment of chronic liver disease. AJR Am J Roentgenol 2009; 193(01):14-27

25 Wagner M, Corcuera-Solano I, Lo G, et al. Technical failure of MR elastography examinations of the liver: experience from a large single-center study. Radiology 2017;284(02):401-412

26 Yin M, Talwalkar JA, Glaser KJ, et al. Dynamic postprandial hepatic stiffness augmentation assessed with MR elastography in patients with chronic liver disease. AJR Am J Roentgenol 2011;197(01):64-70

27 Pepin KM, Chen J, Glaser KJ, et al. MR elastography derived shear stiffness-a new imaging biomarker for the assessment of early tumor response to chemotherapy. Magn Reson Med 2014;71(05): 1834-1840

28 Gordic S, Ayache JB, Kennedy P, et al. Value of tumor stiffness measured with MR elastography for assessment of response of hepatocellular carcinoma to locoregional therapy. Abdom Radiol (NY) 2017;42(06):1685-1694
29 Abramson RG, Arlinghaus LR, Dula AN, et al. MR imaging biomarkers in oncology clinical trials. Magn Reson Imaging Clin N Am 2016;24(01):11-29

30 Blachier M, Leleu H, Peck-Radosavljevic M, Valla DC, RoudotThoraval F. The burden of liver disease in Europe: a review of available epidemiological data. J Hepatol 2013;58(03):593-608

31 Bhala N, Angulo P, van der Poorten D, et al. The natural history of nonalcoholic fatty liver disease with advanced fibrosis or cirrhosis: an international collaborative study. Hepatology 2011;54(04):1208-1216

32 Noureddin M, Lam J, Peterson MR, et al. Utility of magnetic resonance imaging versus histology for quantifying changes in liver fat in nonalcoholic fatty liver disease trials. Hepatology 2013;58(06):1930-1940

33 Serai SD, Dillman JR, Trout AT. Proton density fat fraction measurements at 1.5- and 3-T hepatic MR imaging: same-day agreement among readers and across two imager manufacturers. Radiology 2017;284(01):244-254

34 Chen J, Talwalkar JA, Yin M, Glaser KJ, Sanderson SO, Ehman RL. Early detection of nonalcoholic steatohepatitis in patients with nonalcoholic fatty liver disease by using MR elastography. Radiology 2011;259(03):749-756

35 Yin M, Glaser KJ, Manduca A, et al. Distinguishing between hepatic inflammation and fibrosis with MR elastography. Radiology 2017;284(03):694-705

36 Wood JC, Enriquez C, Ghugre N, et al. MRI R2 and R2* mapping accurately estimates hepatic iron concentration in transfusiondependent thalassemia and sickle cell disease patients. Blood 2005;106(04):1460-1465

37 Berdoukas V, Chouliaras G, Moraitis P, Zannikos K, Berdoussi E, Ladis $\mathrm{V}$. The efficacy of iron chelator regimes in reducing cardiac and hepatic iron in patients with thalassaemia major: a clinical observational study. J Cardiovasc Magn Reson 2009;11:20

38 Westwood M, Anderson LJ, Firmin DN, et al. A single breath-hold multiecho $\mathrm{T}^{*}$ cardiovascular magnetic resonance technique for diagnosis of myocardial iron overload. J Magn Reson Imaging 2003;18(01):33-39

39 Alústiza Echeverría JM, Castiella A, Emparanza JI. Quantification of iron concentration in the liver by MRI. Insights Imaging 2012;3 (02):173-180

40 Wahidiyat PA, Liauw F, Sekarsari D, Putriasih SA, Berdoukas V, Pennell DJ. Evaluation of cardiac and hepatic iron overload in thalassemia major patients with $\mathrm{T}^{*}$ magnetic resonance imaging. Hematology 2017;22(08):501-507

41 Gandon Y, Olivié D, Guyader D, et al. Non-invasive assessment of hepatic iron stores by MRI. Lancet 2004;363(9406):357-362 